\title{
Characterizing Heparin Tetrasaccharides Binding to Amyloid-Beta Peptide
}

\author{
Xiang Zhou ${ }^{1 \dagger}$, Yuanyuan Wang ${ }^{2 \dagger}$, Wei Zheng ${ }^{2}$, Guangxiu Deng ${ }^{1}$, Fuyi Wang ${ }^{3 *}$ and Lan Jin ${ }^{1 *}$ \\ ${ }^{1}$ National Glycoengineering Research Center, Shandong Key Laboratory of Carbohydrate Chemistry and Glycobiology, \\ Shandong University, Qingdao, China, ${ }^{2}$ Beijing National Laboratory for Molecular Sciences, CAS Key Laboratory of Analytical \\ Chemistry for Living Biosystems Institute of Chemistry, Chinese Academy of Sciences, University of Chinese Academy of \\ Sciences, Beijing, China, ${ }^{3}$ College of Traditional Chinese Medicine, Shandong University of Traditional Chinese Medicine, Jinan, \\ China
}

The aggregation of $\beta$-amyloid peptide (AB) is one potential cause for Alzheimer's disease (AD). Heparin can either promote or inhibit $A \beta$ aggregation. The sulfation pattern and chain size determine its binding affinity and its role. Using 2D-NMR analysis and molecular modelling, the binding motif of heparin oligoaccharides to $A \beta$ was determined to be HexA-GlcNS-IdoA2SGlcNS6S. Iduronic acid epimerization and 6-O-sulfation are key factors for the binding affinity, while 3-O-sulfation of Arixtra (heparin pentasaccharide) is not involved in the binding to $A \beta$. Hydrogen-deuterium exchange mass spectrometry (HDX-MS) was used to study the glycosaminoglycan (GAG)-peptide complex and identified $\mathrm{V} 12 \mathrm{HHQKL} 17$ as the binding site of GAG at $A \beta$. Furthermore, an MTT assay was applied to evaluate the anti-A $\beta$ fibril formation function of heparin tetrasaccharide, and indicated that the heparin tetrasaccharide with the defined sequence represents a promising inhibitor of $A \beta$ aggregation.

Keywords: heparin, $\beta$-amyloid peptide, interaction, NMR, hydrogen/deuterium exchange mass spectrometry

\section{INTRODUCTION}

Alzheimer's disease (AD) is a neurodegenerative disease that mainly occurs in people over 65 years of age. The number of patients suffering from $\mathrm{AD}$ around the world was 46.8 million in 2015, a number expected to increase rapidly to approximately 131.5 million in 2050 . The currently available treatments for $\mathrm{AD}$ are post-symptomatic and provide limited relief. In recent years, amyloid plaques and Tau pathology have been widely accepted as the two hallmarks of $\mathrm{AD}$, and their explicit mechanisms lead to the development of therapies that target the course of the disease. The amyloid plaques are mainly composed of $\beta$-amyloid peptide $(A \beta)$, which is derived from the amyloid precursor protein and consists of 39-42 amino acid residues (see Supplementary Figure S4 in the Supplementary Material). Under physiological conditions, different forms of $A \beta$, including the nontoxic monomer, toxic and soluble oligomers, and aggregated amyloid fibrils, co-exist in a dynamic equilibrium (Benilova et al., 2012). An excess of $A \beta$ can push the equilibrium to amyloid fibrils, and the fibrils then infringe the nerve cells and potentially increase the risk of AD. Several immunotherapeutic antibodies targeting $\mathrm{A} \beta$ have reached phase II or III clinical trials (Ariga et al., 2010). Several AD related drugs have also been submitted to the Center for Drug Evaluation worldwide. One of them is Aducanumab (Biogen), which has been approved controversially by FDA but rejected by EMA this year, due to its uncertainty of the efficacy. In addition to these protein-based drugs, sulfated oligosaccharides are promising agents to neutralize excess $A \beta$ because they are less costly and capable of passing the blood-brain barrier. However, carbohydrates are usually 
heterogeneous and can bind to many proteins. Their undefined structures and unspecific mechanisms are major challenges for developing carbohydrate-based drugs. Heparin related oligosaccharides are being actively investigated as potential drugs for the treatment of $\mathrm{AD}$, which is possibly the result of the binding between the sulfated oligosaccharides and $A \beta$. However, the detailed binding mechanism between the sulfated oligosaccharides and $\mathrm{A} \beta$ has not been elucidated.

Heparin belongs to the glycosaminoglycan (GAG) family, which includes heparan sulfate (HS), chondroitin sulfate (CS), dermatan sulfate, hyaluronic acid and other subfamilies based on their different repeating disaccharide units and sulfation patterns. GAGs can either promote or inhibit the aggregation of $A \beta$, with the size of the GAG molecules being the key factor. For example, HS polysaccharide accelerates $A \beta$ aggregation, while small heparin oligosaccharides can inhibit and reverse the process of aggregation. (Zhu et al., 2001; Walzer et al., 2002). The reason is that the large GAG can bind to multiple $\mathrm{A} \beta$ monomers and act as a core for the aggregation, while the small oligosaccharides can only bind to single $A \beta$ monomers and hinder the formation of $A \beta \beta$ sheet or peeling $A \beta$ from the fibrils. The negatively charged groups on GAGs are important because of their binding affinity to $A \beta$. For example, when the sulfo groups were removed from HS, it lost its ability to promote A $\beta$ fibril formation (Castillo et al., 1999). Binding of $A \beta$ to heparin/HS oligosaccharides has been studied using solidstate NMR. There were no significant differences of the $\mathrm{K}_{\mathrm{d}}$ values $(\sim 40 \mu \mathrm{M})$ from hexamer to octadecamer derived from heparin degradation (Stewart et al., 2016). N-sulfation or 6-O-sulfation in chemically synthesized HS polysaccharides was essential in binding with $A \beta$ beyond electrostatic attraction (Madine et al., 2012). Study of HS hexasaccharide and decasaccharide obtained by well-defined synthesis in binding with isotopically labeled $A \beta$ indicated the importance of specific amino acids at the C-terminus residues in the A $\beta$ fibrils (Wang et al., 2021). CS disaccharides with different sulfation positions have been shown different binding forces to $A \beta$, with an ordering from strong to weak of 6-O-sulfation, 4-Osulfation, and 2-O-sulfation (Fraser et al., 2001). Conformation of proteins and their interaction with carbohydrates were studied more and more using HDX-MS (Chalmers et al., 2011; Engen, 2009). In this study, we elaborate the roles of each sulfo and carboxyl group of heparin tetrasaccharides and delineate the sequence required for binding to $A \beta$.

\section{MATERIALS AND METHODS}

\subsection{Materials}

Heparin was purchased from Zaozhuang Sinock Biochemical Pharmaceutical Co., Ltd. Heparinase I was purchased from Beijing Adhoc International Technologies Co., Ltd. Fondaparinux injection was obtained from the hospital. A $\beta$ was solid synthesized by ChinaPeptides Co., Ltd. Deuterium oxide $\left(99.9 \% \mathrm{D}_{2} \mathrm{O}, \mathrm{CIL}\right)$ was purchased from Qingdao Tenglong Weibo Technology Co., Ltd. Norell S-5-500-HW-7 NMR tubes were used for the complex samples of heparin oligosaccharides with $\mathrm{A} \beta$. Normal $5 \mathrm{~mm}$ NMR tubes were used for the oligosaccharide structure characterization.

\subsection{Methods}

\subsubsection{Preparation of Heparin Tetrasaccharides}

Heparin was partially digested with heparinase I. The oligosaccharides were fractionated using a GE AKTA purifier 100 system with a Superdex TM Peptide 10/300 GL column. The fractions were eluted with $0.2 \mathrm{M} \mathrm{NH}_{4} \mathrm{HCO}_{3}$ at a flow rate of $0.4 \mathrm{ml} / \mathrm{min}$. The tetrasaccharide fraction was then separated using a Spherisorb S5 SAX $5 \mu \mathrm{m}$ column. A linear gradient of 0-2.0 M $\mathrm{NaCl}$ (pH 3.5) over $2 \mathrm{~h}$ was used. The flow rate was $4 \mathrm{ml} / \mathrm{min}$. UV absorption was monitored at $232 \mathrm{~nm} .{ }^{1} \mathrm{H}$ spectra were used to characterize the purity based on the signal integration.

\subsubsection{Structure Characterization of Oligosaccharides by MS/MS}

MS/MS studies were performed on an ion-trap-time-of-flight (IT-TOF) hybrid mass spectrometer (Shimadzu) in the negative mode. The samples were injected using a syringe in 50:50 (v:v) methanol/water containing $1 \mathrm{mM} \mathrm{NaOH}$. The instrument parameters were set as follows: interface voltage, $-3.5 \mathrm{kV}$; nebulizing gas flow rate, $1.5 \mathrm{~L} / \mathrm{min}$ and collision-induced dissociation energy: $50 \%$. The sequence was elucidated by glycosidic fragmentations and cross-ring fragmentations.

\subsubsection{Structure Characterization of Oligosaccharides by 2D-NMR}

$3 \mathrm{mg}$ of each oligosaccharide was dissolved in $500 \mu \mathrm{L}$ of deuterium oxide $\left(\mathrm{D}_{2} \mathrm{O}\right)$ and lyophilized to remove exchangeable protons. The powder was then re-dissolved in $550 \mu \mathrm{L}$ of $\mathrm{D}_{2} \mathrm{O}$ and transferred into $5 \mathrm{~mm}$ NMR tubes. NMR spectra were acquired at $600 \mathrm{MHz}\left({ }^{1} \mathrm{H}\right)$ or $150 \mathrm{MHz}\left({ }^{13} \mathrm{C}\right)$ with a Bruker AVANCE III $600 \mathrm{MHz}$ spectrometer equipped with a $5 \mathrm{~mm}$ cryoprobe. Chemical shifts were recorded with reference to the HDO solvent signal at $298 \mathrm{~K}$. 2D ${ }^{1} \mathrm{H}-{ }^{1} \mathrm{H}$ COSY, ${ }^{1} \mathrm{H}_{-}{ }^{13} \mathrm{C}$ HSQC, and ${ }^{1} \mathrm{H}_{-}{ }^{13} \mathrm{C}$ HMBC were acquired with standard pulse sequences. Cross peaks in the HSQC spectra of each oligosaccharide were assigned and labeled.

\subsubsection{Molecular Docking and Modeling Simulations}

Molecular docking and modeling were performed using AutoDock 4.2.6, a fully automatic docking program available open source. The solid-state NMR structure of $A \beta$ was retrieved from the Protein Data Bank (PDB) under code 6TI5 (Cerofolini et al., 2020), which is a fibril of hexadecylmer, while an NMRoptimized structure was used for tetrasaccharide 1 (Jin et al., 2009). All of the hydrogen atoms were added to $A \beta$ fibril and Gasteiger charge was used. A volume of $(120,120,120)$ grid points with $0.514 \AA$ spacing was used. $A \beta$ was put in the center of the cube larger enough to cover the whole surface of $A \beta$. The tetrasaccharide 1 was placed randomly into the box. During the whole docking process, all monosaccharide rings were fixed at their starting conformations, while the ring substituents were defined as flexible and could be rotated freely. Genetic Algorithm was used for docking.

\subsubsection{GMSA Assay}

The GMSA assay was performed according to procedures previously described. Briefly, $1 \mathrm{mg} \mathrm{A} \beta$ was dissolved in $500 \mu \mathrm{L}$ 
$\mathrm{NaAc}(20 \mathrm{mM}, \mathrm{pH}$ 3.5, precooled in ice) without any disaggregation process and the final concentration of $2 \mu \mathrm{g} / \mu \mathrm{L}$ $\mathrm{A} \beta$ was used thereafter. 2-aminoacridone-labeled tetrasaccharides were combined with $A \beta$ in a certain molar ratio for $15 \mathrm{~min}$ at room temperature in a total volume of $20 \mu \mathrm{L}$. They were then loaded in wells of $1 \%$ agarose gel in $10 \mathrm{mM}$ Tris- $\mathrm{HCl}(\mathrm{pH} \mathrm{7.4)}$ and $1 \mathrm{mM}$ EDTA. Electrophoresis was performed at $100 \mathrm{~V}$ for $20 \mathrm{~min}$ in a horizontal agarose electrophoresis system using an electrophoresis buffer comprising $40 \mathrm{mM}$ Tris/acetic acid ( $\mathrm{pH} \mathrm{8.0)}$ and $1 \mathrm{mM}$ EDTA. The fluorescent oligosaccharides were visualized on a FluorChem $\mathrm{Q}$ gel analysis system.

\subsubsection{HDX-MS Analysis}

An in-house system was set up using a Waters Acquity-H-CLASS UPLC and Xevo G2 Q-TOF mass spectrometer. A refrigerator was used to cool the injector, loop, columns, 6-way valve and switch valve. $A \beta$ was treated in TFA and HFIP to break up any possible preexisting aggregates. The complex of $\mathrm{A} \beta$ and tetrasaccharide 1 was incubated in $\mathrm{H}_{2} \mathrm{O}(\mathrm{pH} 3.5)$ at $4^{\circ} \mathrm{C}$ for $120 \mathrm{~min}$. Nine volumes of $\mathrm{D}_{2} \mathrm{O}(\mathrm{pH} 3.5)$ were added to initiate the HDX. After $10 \mathrm{~min}, 1 \mu \mathrm{L}$ of $10 \%$ ice-cold formic acid (FA) solution ( $\mathrm{pH}$ 2.5) was added to quench the HDX. The deuterated $\mathrm{A} \beta$ was loaded onto an on-line pepsin proteolytic column. After digestion, the valve was switched, and the peptides flowed into a C18 column $(5 \mu \mathrm{m}, 2.1 \times 50 \mathrm{~mm})$. The mobile phase A was $0.1 \% \mathrm{FA}$, and mobile $\mathrm{B}$ was $0.1 \% \mathrm{FA}$ in acetonitrile. The elution gradient was $20-60 \%$ mobile phase B in $6 \mathrm{~min}$. The flow rate was $200 \mu \mathrm{L} / \mathrm{min}$. The mass spectrometer parameters were set as follows: capillary voltage, $3 \mathrm{kV}$; source temperature, $100^{\circ} \mathrm{C}$; desolvation temperature, $350^{\circ} \mathrm{C}$. The spectra were acquired in MSe mode with low collision energy of $6 \mathrm{~V}$ and high collision energy of $25-50 \mathrm{~V}$.

\subsubsection{MTT Assay}

The SH-SY5Y and PC 12 cell lines were cultured separately in Dulbecco's modified Eagle's medium supplemented with 10\% fetal bovine serum at $37^{\circ} \mathrm{C}, 5 \% \mathrm{CO}_{2}$ in an incubator. The cells $(4,000$ or 6,000 cells/well) were seeded in 96-well plates and cultured in DMEM with $10 \%$ FBS. The culture medium was changed to fresh medium supplemented with $2 \mu \mathrm{MA} \beta$ peptide alone or with tetrasaccharide 1 at concentrations of 10,50 or $100 \mu \mathrm{g} / \mathrm{ml}$. After 24 or $36 \mathrm{~h}$ incubation, $20 \mu \mathrm{l}$ of $5 \mathrm{mg} / \mathrm{ml} \mathrm{MTT}$ was added. After $4 \mathrm{~h}$ incubation at $37^{\circ} \mathrm{C}$, the supernatant was discarded, and $150 \mu \mathrm{L}$ of DMSO was added. Enzyme-linked immunoassay was used to measure the absorbance value at $570 \mathrm{~nm}$ for the determination wavelength and $630 \mathrm{~nm}$ for the reference wavelength. Statistical significance was established by SPS 17.0 statistical software, and single factor analysis of variance and $p$-values were represented as follows: ${ }^{*} p<0.05$ and ${ }^{* *} p<0.01$. The experimental results were in the form of the mean and standard deviation.

2.2.8 Interaction of $A \beta$ With Heparin Oligosaccharides Heparin oligosaccharide was dissolved in $\mathrm{D}_{2} \mathrm{O}$, into which $\mathrm{A} \beta$ was added, and the supernatant was transferred into the NMR tube.
Complexes of heparin oligosaccharides and $\mathrm{A} \beta$ were prepared with a molar ratio of $1: 1$ in $120 \mu \mathrm{L}$ of $\mathrm{D}_{2} \mathrm{O} .{ }^{1} \mathrm{H}_{-}{ }^{13} \mathrm{C}$ HSQC spectra were acquired.

\section{RESULTS}

\subsection{Preparation of Heparin Tetrasaccharides}

Three heparin tetrasaccharides 1-3 with different sulfation patterns were prepared by partial enzymatic digestion of heparin, gel permeation chromatography fractionation, and strong anion-exchange liquid chromatography separation (Supplementary Figure S1). ${ }^{1} \mathrm{H}$ spectra were used to characterize the purity based on the signal integration. (Supplementary Figure S3). The impurities were labelled as asterisks and the amount of which were up to $12.2 \%$.

\subsection{Structure Characterization of Oligosaccharides by MS/MS and 2D-NMR}

The structures were determined by tandem MS and 2D-NMR (Supplementary Figures S2, S4). Tetrasaccharide 1, $\Delta$ UA2SGlcNS6S-IdoA2S-GlcNS6S, is the fully sulfated form, i.e., substituted by three sulfo groups per disaccharide. Tetrasaccharide 2, $\Delta$ UA2S-GlcNS6S-GlcA-GlcNS6S, is the 2$\mathrm{O}$-desulfated and epimerized form of 1 , and tetrasaccharide 3, $\triangle \mathrm{UA2S}-$ GlcNS6S-IdoA2S-GlcNS, is the 6-O-desulfated form of 1 (Figure 1).

\subsection{Molecular Docking and Modeling Simulations}

The conformation with the lowest binding energy (Figure 2) was examined using the Pymol software to evaluate the degree of ionic interaction between specific sulfo and carboxyl groups of tetrasaccharide 1 and the basic amino acid residues of $A \beta$. The $\mathrm{A} \beta$ fibril was a hexadecylmer in an anti-parallel construction, composed by eight chains (A-H chains) on each side. And four chains (B-E) interacted with one tetrasacchride 1 mainly through ionic attraction and hydrogen bonds. These interactions involved all His-14 and Lys-16 in B-E chains.

\subsection{GMSA Assay}

A gel mobility shift assay (GMSA) was used to evaluate the binding affinities of tetrasacchrides $1-3$ to A $\beta$ containing 40 amino acid residues. The tetrasaccharides were derived by 2 aminoacridone, incubated with $\mathrm{A} \beta$, co-electrophoresed, and detected by fluorescence on a FluorChem $\mathrm{Q}$ gel analysis system (Figure 3). The tetrasaccharide- $A \beta$ complexes showed slower motilities than their corresponding controls of tetrasaccharide alone. Tetrasaccharide 1 showed the strongest binding affinity, as most of it was withheld by $A \beta$ at the sample loading position, and only a faint band corresponding to the unbound format was observed. Tetrasaccharide 3 exhibited much weaker binding affinity than 1 , while tetrasaccharide 2 bonded most weakly to $A \beta$. 

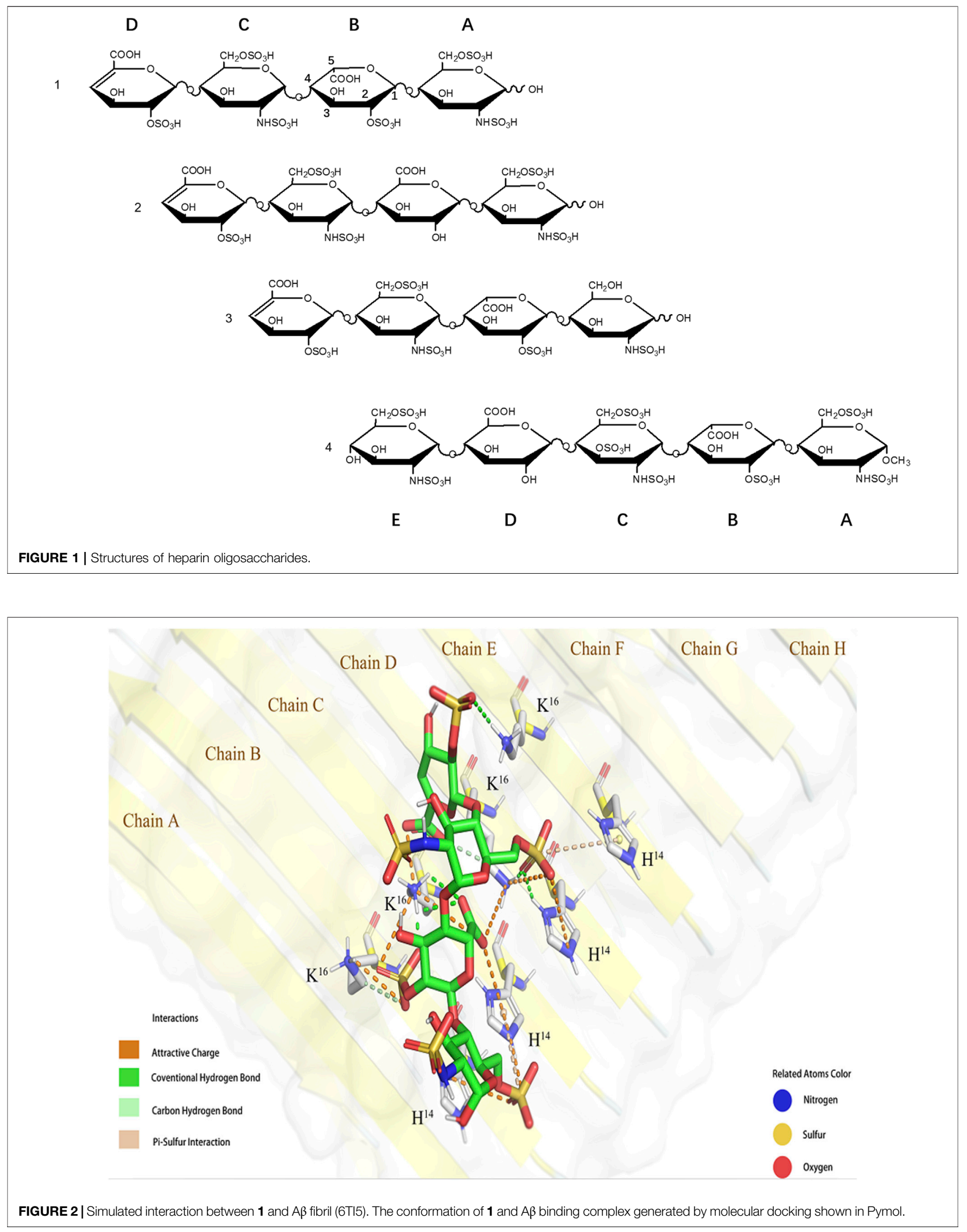

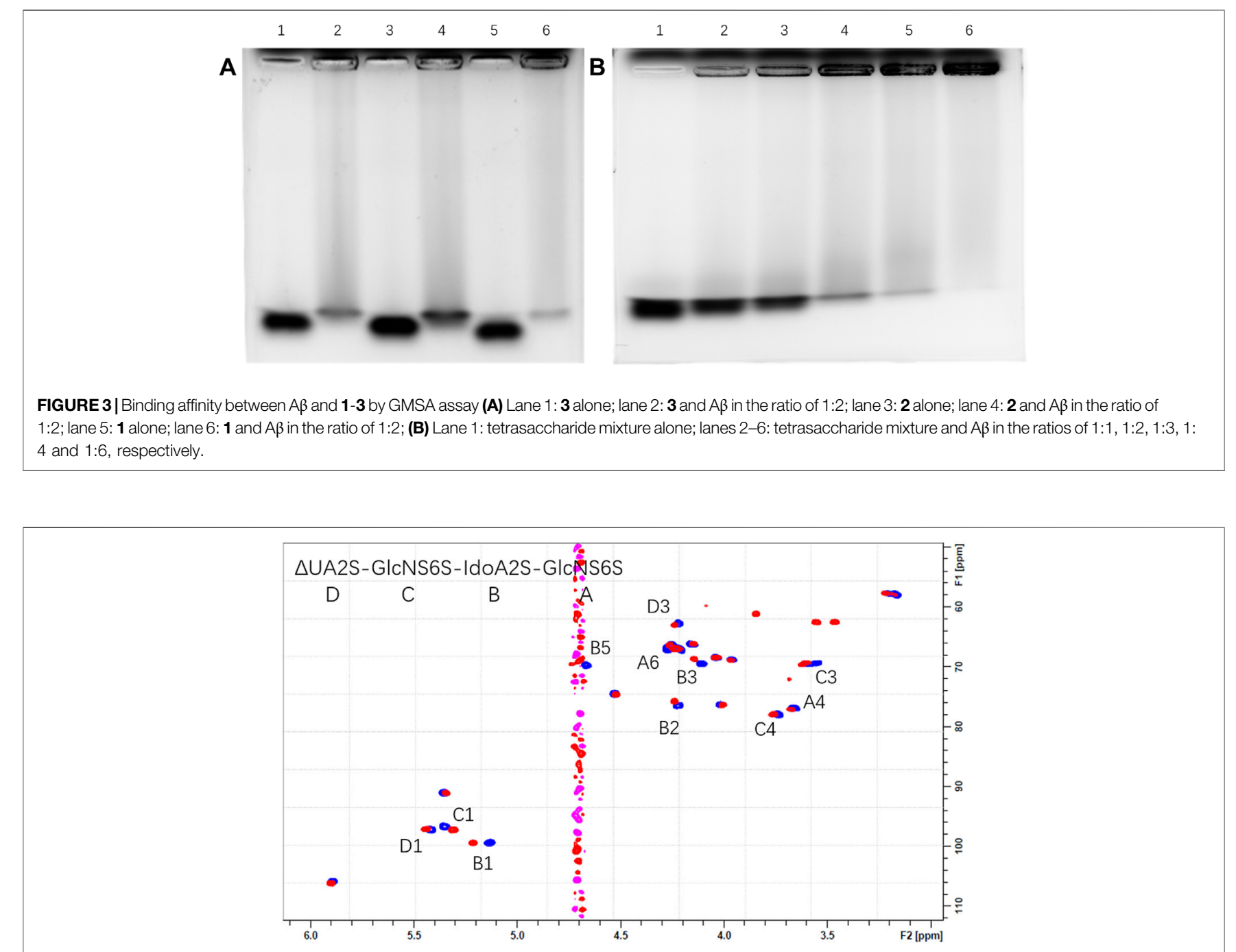

FIGURE $\mathbf{4}$ | The overlaid ${ }^{1} \mathrm{H}^{-13} \mathrm{C}$ HSQC spectra of $\mathbf{1}$ only (blue) and $\mathbf{1}$ with $\mathrm{A} \beta$ (pink).

\subsection{Interaction of $A \beta$ With Heparin Oligosaccharides}

The overlaid HSQC spectra of all oligosaccharides 1-4 only and with A $\beta$ were shown in Supplementary Figure S6. Obvious chemical shift perturbations of certain cross peaks upon the binding were observed after the addition of $A \beta$. The results indicated that the 3-Osulfo group did not contribute to the binding but did decrease the affinity, which could be due to the stereo-hindrance effect. (Figure 4 and Supplementary Figure S6).

\subsection{HDX-MS Analysis}

The deuterated $A \beta$ was loaded onto an on-line pepsin proteolytic column for digestion. Four proteolytic peptides, designated T1, $\mathrm{T} 2$, $\mathrm{T} 3$, and $\mathrm{T} 4$, derived from peptic digestion of $\mathrm{A} \beta$ were detected to cover the full sequence of $A \beta$ (Figure 5). The changes of deuterium incorporation for each peptic peptide subject to incubation of $\mathrm{A} \beta$ in $\mathrm{D} 2 \mathrm{O}$ for different times were monitored by MS. The data were analyzed by HX-Express software based on the centroid of the molecular ion isotope peaks.

\subsection{MTT Assay}

The inhibitory activities of tetrasaccharide 1 against $A \beta$ fibril formation were assessed by an MTT assay using neurocyte cell lines SH-SY5Y and PC12, respectively (Figure 6). The viability of neurocytes decreased significantly after exposure to $2 \mu \mathrm{M}$ aged $\mathrm{A} \beta$ for 24 and $36 \mathrm{~h}$, respectively. The toxicity of $\mathrm{A} \beta$ was attenuated when tetrasaccharide 1 was added to the cells. Addition of tetrasaccharide 1 at $100 \mu \mathrm{g} / \mathrm{ml}$ could significantly reduce the cell injuries caused by $A \beta$. This effect indicated that the interaction between tetrasaccharide 1 and $A \beta$ can inhibit the formation of the toxic oligomers or aggregates.

\section{DISCUSSION}

The negatively charged groups on the GAG oligosaccharides are responsible for its affinity to $A \beta$. The sulfo groups of heparin and HS can be sorted to 2-O-sulfo of the uronic acid residue, 3- and 6$\mathrm{O}$-sulfo at the GlcN residue, and $\mathrm{N}$-sulfo of the $\mathrm{GlcN}$ residue. 

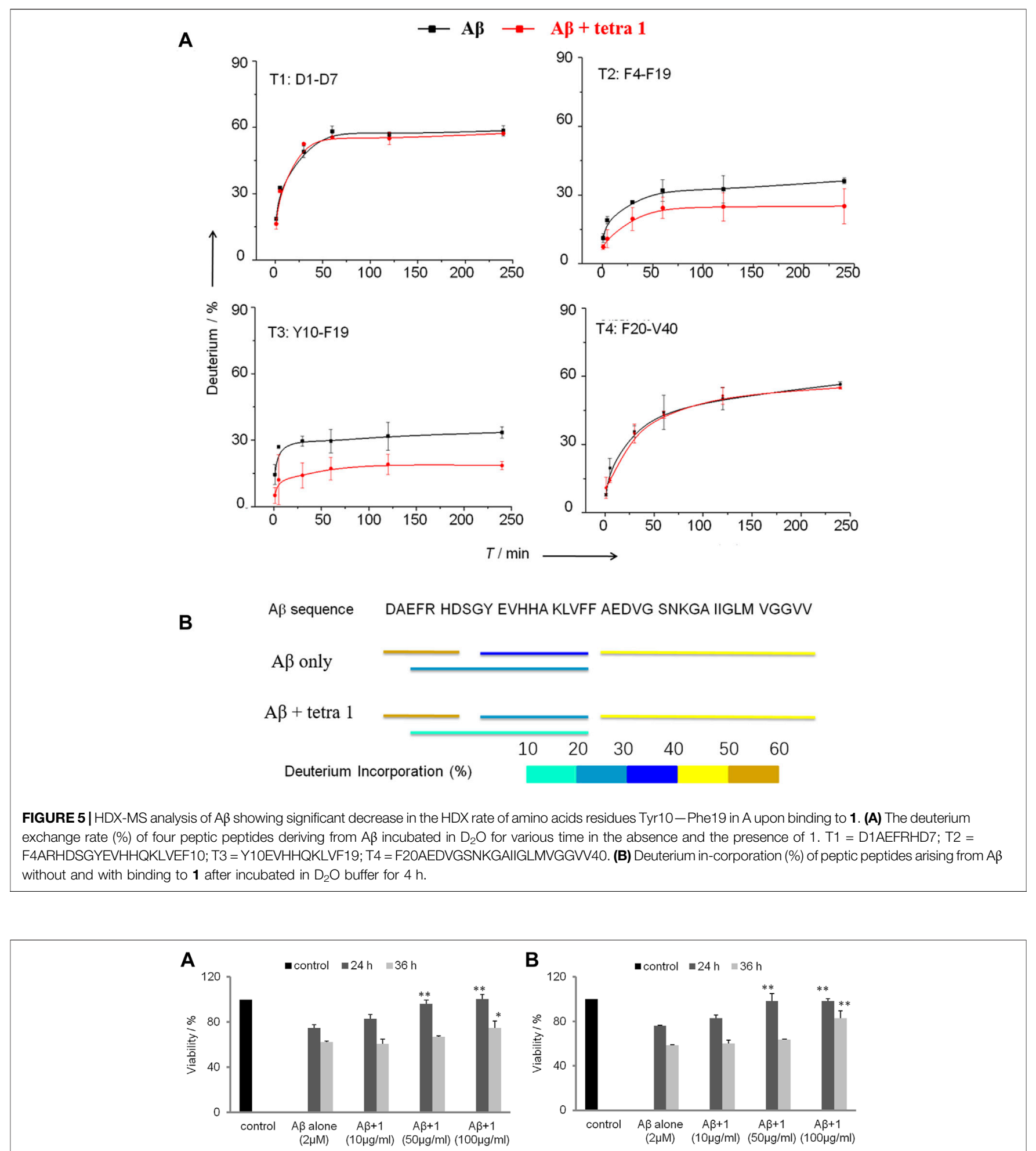

FIGURE 6 | The inhibition effect of $\mathbf{1}$ against the cytotoxicity caused by A $\beta$. (A) SH-SY5Y cells and (B) PC12 cells after 24 and 36 h $\left({ }^{*} p<0.05,{ }^{\star \star} p<0.01\right)$.

GMSA results suggest that the 2-O-sulfo group at the uronic acid residue and 6-O-sulfo group at the terminal residue are involved in the interaction with $\mathrm{A} \beta$. The docking simulation suggested that the His-14 to Lys-16 regions of $A \beta$ fibril (B-E chains) were involved in binding with tetrasaccharide 1 . The IdoA2S residue was in the ${ }^{1} \mathrm{C}_{4}$ conformation, and both carboxyl group 
and 2-O-sulfo group were in closer contact with $\mathrm{A} \beta$. IdoA2S was electrostatically attracted by His-14 (C chain) and Lys-16 (B-D chains) of $A \beta$, and was the essential motif in binding. It is worth mentioning that the 6-O-sulfo group at the reducing end is attracted by His-14 in the $B$ and $C$ chains of $A \beta$, which explains why tetrasaccharide 2 also bonded to $A \beta$ to some degree in the GMSA experiment.

In NMR analysis, the synthetic $A \beta$ served as a probe to elucidate the essential binding regions of 1 . As shown in Figure 4, GlcNS (A2, C1, C2, C3), 6S at the reducing end (A6S), IdoA2S (B1, B2, B3, B5) and the carboxyl group of the non-reducing end (D4) were crucial to the binding. Obvious chemical shift perturbations of certain cross peaks upon the binding were observed after the addition of $A \beta$. The internal monosaccharide rings -GlcNS6S-IdoA2S- (C-B) were involved in the binding interaction, with the IdoA2S showing the largest chemical shift perturbations, suggesting that it plays an essential role in binding with $\mathrm{A} \beta$. By combining the molecular docking and HSQC-NMR results, the binding sequence for heparin tetrasaccharides to bind to $A \beta$ could be deduced to be HexA-GlcNS-IdoA2S-GlcNS6S. The conformation of the internal hexauronic acid residue and the proper orientation of the substituents were the determinants. In the linear structure of heparin, in addition to the 2-O-sulfation at the uronic acid residue and 6-Osulfation and $\mathrm{N}$-sulfation at the GlcN residue included in 1 , the 3-O-sulfation at the GlcN residue occasionally occurs and is responsible for the anticoagulant activity of heparin. The 3O-sulfation in Arixtra ( 4 in Figure 1) did not contribute to the binding with $\mathrm{A} \beta$ based on the HSQC spectrum, but IdoA2S was still involved in binding. And this could be due to the stereo hindrance effect.

Based on the HDX-MS results, when 1 was present, the peptides T2 and T3 showed a significant decrease of deuterium update rate, while the rates of peptides $\mathrm{T} 1$ and T4 remained almost unchanged (Figure 5). These results provide evidence that the Tyr10-Phe19 region participates in the interaction between $A \beta$ and 1 , in consistent with prediction by docking simulation. Cardin and Weintraub (Cardin and Weintraub, 1989) proposed a consensus sequence $\mathrm{XBBXBX}$ (where $\mathrm{B}$ is a basic amino acid residue and $X$ is any amino acid residue) in proteins to be the binding site to GAGs. Nguyen (Nguyen and Rabenstein, 2016) confirmed the binding affinity of $A \beta 12-18$ fragment to heparin decreased dramatically if the amino acid residue His-13 or His-14 was replaced by alanine. Therefore, the minimum sequence on $\mathrm{A} \beta$ that is required for its binding to heparin tetrasaccharide should be V12HHQKL17.

Biomolecule-based drugs are becoming a major force in the pharmaceutical industry. However, unlike the boost in protein-

\section{REFERENCES}

Ariga, T., Miyatake, T., and Yu, R. K. (2010). Role of Proteoglycans and Glycosaminoglycans in the Pathogenesis of Alzheimer's Disease and Related based drugs, the development of carbohydrate-based drugs is somewhat frustrated, mainly because of their heterogeneous structures and unspecific or unclear functions. Heparin oligosaccharides exhibited efficient anti-A $\beta$ aggregation activity. However, these negatively charged and heterogonous carbohydrates are known to bind hundreds of endogenous proteins, which increases the risk of many side effects such as bleeding. GAGs are proven to have defined sequences. (Ly et al., 2011). Much as the discovery of a specific anti-thrombin pentasaccharide sequence in heparin has led to discovery of Arixtra, a successful synthetic anticoagulant drug. The $\mathrm{A} \beta$ binding sequence of heparin oligosaccharides elucidated herein provides a promising template for chemical or bio-synthesis of therapeutic agents for anti-A $\beta$ aggregation. It could minimize undesired side effects while retaining $A \beta$ binding activity and cross the blood-brain barrier. In addition, combining with efficient biosynthesis of GAGs (Xu et al., 2011) will accelerate the breakthrough of carbohydrate drugs in the new era of health care.

\section{DATA AVAILABILITY STATEMENT}

The raw data have been deposited on Mendeley Data, and publicly available at https://data.mendeley.com/datasets/ zmrvtt2296/1.

\section{AUTHOR CONTRIBUTIONS}

$\mathrm{XZ}$ and YW contributed equally to this work in MS. WZ contributed to the simulation. GD contributed to the preparation of heparin oligosaccharides. LJ contributed to NMR. FW, and LJ contributed to the study design and wrote the manuscript. All authors read and approved the final manuscript.

\section{FUNDING}

This study is financially supported by Natural Science Foundation of China (21302113) and Beijing National Laboratory for Molecular Sciences (BNLMS).

\section{SUPPLEMENTARY MATERIAL}

The Supplementary Material for this article can be found online at: https://www.frontiersin.org/articles/10.3389/fmolb.2022.824146/ full\#supplementary-material

Disorders: Amyloidogenesis and Therapeutic Strategies-A Review. J. Neurosci. Res. 88 (11), 2303-2315. doi:10.1002/jnr.22393

Benilova, I., Karran, E., and De Strooper, B. (2012). The Toxic A $\beta$ Oligomer and Alzheimer's Disease: an Emperor in Need of Clothes. Nat. Neurosci. 15 (3), 349-357. doi:10.1038/nn.3028 
Cardin, A. D., and Weintraub, H. J. (1989). Molecular Modeling of ProteinGlycosaminoglycan Interactions. Arteriosclerosis 9, 21-32. doi:10.1161/01. ATV.9.1.21

Castillo, G. M., Lukito, W., Wight, T. N., and Snow, A. D. (1999). The Sulfate Moieties of Glycosaminoglycans Are Critical for the Enhancement of $\beta$ Amyloid Protein Fibril Formation. J. Neurochem. 72 (4), 1681-1687. doi:10. 1046/j.1471-4159.1999.721681.x

Cerofolini, L., Ravera, E., Bologna, S., Wiglenda, T., Böddrich, A., Purfürst, B., et al. (2020). Mixing $A \beta(1-40)$ and $A \beta(1-42)$ Peptides Generates Unique Amyloid Fibrils. Chem. Commun. 56 (62), 8830-8833. doi:10.1039/D0CC02463E

Chalmers, M. J., Busby, S. A., Pascal, B. D., West, G. M., and Griffin, P. R. (2011). Differential Hydrogen/deuterium Exchange Mass Spectrometry Analysis of Protein-Ligand Interactions. Expert Rev. Proteomics 8 (1), 43-59. doi:10.1586/ epr.10.109

Engen, J. R. (2009). Analysis of Protein Conformation and Dynamics by Hydrogen/ Deuterium Exchange MS. Anal. Chem. 81 (19), 7870-7875. doi:10.1021/ ac901154s

Fraser, P. E., Darabie, A. A., and McLaurin, J. (2001). Amyloid- $\beta$ Interactions with Chondroitin Sulfate-Derived Monosaccharides and Disaccharides. J. Biol. Chem. 276 (9), 6412-6419. doi:10.1074/jbc.M008128200

Jin, L., Hricovini, M., Deakin, J. A., Lyon, M., and Uhrin, D. (2009). Residual Dipolar Coupling Investigation of a Heparin Tetrasaccharide Confirms the Limited Effect of Flexibility of the Iduronic Acid on the Molecular Shape of Heparin. Glycobiology 19 (11), 1185-1196. doi:10.1093/glycob/cwp105

Ly, M., Leach, F. E., III, Laremore, T. N., Toida, T., Amster, I. J., and Linhardt, R. J. (2011). The Proteoglycan Bikunin Has a Defined Sequence. Nat. Chem. Biol. 7 (11), 827-833. doi:10.1038/nchembio.673

Madine, J., Pandya, M. J., Hicks, M. R., Rodger, A., Yates, E. A., Radford, S. E., et al. (2012). Site-Specific Identification of an A $\beta$ Fibril-Heparin Interaction Site by Using Solid-State NMR Spectroscopy. Angew. Chem. Int. Ed. 51 (52), 13140-13143. doi:10.1002/anie.201204459

Nguyen, K., and Rabenstein, D. L. (2016). Interaction of the Heparin-Binding Consensus Sequence of $\beta$-Amyloid Peptides with Heparin and HeparinDerived Oligosaccharides. J. Phys. Chem. B 120 (9), 2187-2197. doi:10.1021/ acs.jpcb.5b12235
Stewart, K. L., Hughes, E., Yates, E. A., Akien, G. R., Huang, T.-Y., Lima, M. A., et al. (2016). Atomic Details of the Interactions of Glycosaminoglycans with Amyloid- $\beta$ Fibrils. J. Am. Chem. Soc. 138 (27), 8328-8331. doi:10.1021/jacs. $6 \mathrm{~b} 02816$

Walzer, M., Lorens, S., Hejna, M., Fareed, J., Hanin, I., Cornelli, U., et al. (2002). Low Molecular Weight Glycosaminoglycan Blockade of $\beta$-amyloid Induced Neuropathology. Eur. J. Pharmacol. 445 (3), 211-220. doi:10.1016/S00142999(02)01759-4

Wang, P., Zhao, J., Hossaini Nasr, S., Otieno, S. A., Zhang, F., Qiang, W., et al. (2021). Probing Amyloid $\beta$ Interactions with Synthetic Heparan Sulfate Oligosaccharides. ACS Chem. Biol. 16 (10), 1894-1899. doi:10.1021/ acschembio.0c00904

Xu, Y., Masuko, S., Takieddin, M., Xu, H., Liu, R., Jing, J., et al. (2011). Chemoenzymatic Synthesis of Homogeneous Ultralow Molecular Weight Heparins. Science 334, 498-501. doi:10.1126/science.1207478

Zhu, H., Yu, J., and Kindy, M. S. (2001). Inhibition of Amyloidosis Using LowMolecular-Weight Heparins. Mol. Med. 7, 517-522. doi:10.1007/BF03401857

Conflict of Interest: The authors declare that the research was conducted in the absence of any commercial or financial relationships that could be construed as a potential conflict of interest.

Publisher's Note: All claims expressed in this article are solely those of the authors and do not necessarily represent those of their affiliated organizations, or those of the publisher, the editors and the reviewers. Any product that may be evaluated in this article, or claim that may be made by its manufacturer, is not guaranteed or endorsed by the publisher.

Copyright (c) 2022 Zhou, Wang, Zheng, Deng, Wang and Jin. This is an open-access article distributed under the terms of the Creative Commons Attribution License (CC $B Y)$. The use, distribution or reproduction in other forums is permitted, provided the original author(s) and the copyright owner(s) are credited and that the original publication in this journal is cited, in accordance with accepted academic practice. No use, distribution or reproduction is permitted which does not comply with these terms. 